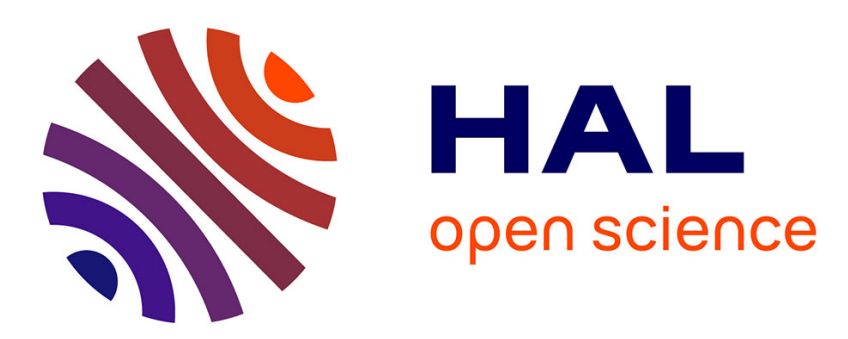

\title{
Cyclable and non-volatile electric field control of magnetism in BiFeO 3 based magnetoelectric heterostructures
}

Christophe Daumont, Jérôme Wolfman, Cecile Autret, Pascal Andreazza, Béatrice Negulescu

\section{To cite this version:}

Christophe Daumont, Jérôme Wolfman, Cecile Autret, Pascal Andreazza, Béatrice Negulescu. Cyclable and non-volatile electric field control of magnetism in BiFeO 3 based magnetoelectric heterostructures. Applied Physics Letters, 2018, 112 (11), pp.112401. 10.1063/1.5018455 . hal01741590

\author{
HAL Id: hal-01741590 \\ https://hal.science/hal-01741590
}

Submitted on 21 Feb 2022

HAL is a multi-disciplinary open access archive for the deposit and dissemination of scientific research documents, whether they are published or not. The documents may come from teaching and research institutions in France or abroad, or from public or private research centers.
L'archive ouverte pluridisciplinaire HAL, est destinée au dépôt et à la diffusion de documents scientifiques de niveau recherche, publiés ou non, émanant des établissements d'enseignement et de recherche français ou étrangers, des laboratoires publics ou privés. 
Cyclable and non-volatile electric field control of magnetism in $\mathrm{BiFeO}_{3}$ based magnetoelectric heterostructures

C. Daumont, J. Wolfman, C. Autret-Lambert, P. Andreazza, and B. Negulescu

Citation: Appl. Phys. Lett. 112, 112401 (2018); doi: 10.1063/1.5018455

View online: https://doi.org/10.1063/1.5018455

View Table of Contents: http://aip.scitation.org/toc/apl/112/11

Published by the American Institute of Physics

Scilight

Sharp, quick summaries illuminating the latest physics research 


\title{
Cyclable and non-volatile electric field control of magnetism in $\mathrm{BiFeO}_{3}$ based magnetoelectric heterostructures
}

\author{
C. Daumont, ${ }^{1}$ J. Wolfman, ${ }^{1}$ C. Autret-Lambert, ${ }^{1}$ P. Andreazza, ${ }^{2}$ and B. Negulescu ${ }^{1, a)}$ \\ ${ }^{1}$ GREMAN, UMR 7347, Université de Tours, INSA Centre Val de Loire, CNRS, Tours, France \\ ${ }^{2}$ ICMN, UMR 7374, Université d'Orléans, CNRS, Orléans, France
}

(Received 7 December 2017; accepted 27 February 2018; published online 12 March 2018)

\begin{abstract}
Room temperature manipulation of the ferromagnetic state via an electric field is investigated in $\mathrm{Ni} /$ $\mathrm{BiFe}_{0.95} \mathrm{Mn}_{0.05} \mathrm{O}_{3}$ thin film heterostructures. A $600 \%$ increase in the magnetic coercive field of the $\mathrm{Ni}$ layer is observed at the initial DC electrical poling of the ferroelectric $\mathrm{BiFe}_{0.95} \mathrm{Mn}_{0.05} \mathrm{O}_{3}$ layer. The magnetoelectric effect is remanent, and the magnetic coercive field can be modulated between a low value and a high value by successively switching the ferroelectric polarization. After the initial poling, the coercive field difference is decreased by subsequent back and forth switching. However, the magnetic bi-stability is preserved at least up to 250 cycles, which is promising for spintronic applications. Published by AIP Publishing. https://doi.org/10.1063/1.5018455
\end{abstract}

Electric field control of magnetism has been intensively studied during the past decade due to its importance for future low power consumption spintronic devices. From the material point of view, multiferroics displaying coupled ferroic order parameters appear as natural candidates. However, there are only a few single phase multiferroic materials at room temperature and their magnetic properties are limited to antiferromagnetic or weak ferromagnetic orders. Technological applications will rather require the use of magnetoelectric heterostructures combining ferromagnetic and piezoelectric/ferroelectric materials. In such heterostructures, depending on the combination of the material properties, three mechanisms leading to the electrical manipulation of magnetism have been identified, namely, strain mediated control of magnetism, electrical charge mediated control of magnetism, and interlayer exchange coupling. ${ }^{1-3}$

Strain mediated control of magnetism was demonstrated in several types of heterostructures, generally using bulk piezoelectric materials such as $\mathrm{PZT},{ }^{4} \mathrm{PMN}-\mathrm{Pt},{ }^{5}$ or $\mathrm{BaTiO}_{3}{ }^{6}$ The strain mostly affects the magnetic anisotropy axis through the coupling of the inverse piezoelectric and magnetostriction effects. It was shown that in this case, both strain and magnetization major loops as a function of the electric field display a similar butterfly shape. ${ }^{1-3}$ In charge-mediated control of magnetism, the spin dependence of the Thomas Fermi screening length generates at the ferromagnetic/ferroelectric interface a modification of both the magnetic anisotropy and the saturation magnetization which depends on the electrical polarization direction. ${ }^{1,3,7}$ As another charge related mechanism, a magneto-ionic control of the interfacial magnetism via migration of the oxidation front through redox reaction was observed recently for the $\mathrm{GdO}_{\mathrm{x}} / \mathrm{Co}$ system, ${ }^{8,9}$ through $\mathrm{Li}^{+}$migration in $\mathrm{Pt} / \mathrm{LiFe}_{5} \mathrm{O}_{8-\delta} / \mathrm{SrRuO}_{3}$ (Ref. 10) or oxygen vacancy migration in $\mathrm{Ti} / \mathrm{ZnO} / \mathrm{SrRuO}_{3}$ heterostructures. ${ }^{11}$

The exchange coupling mechanism will occur when the ferroelectric phase is also antiferromagnetic and it manifests through a shift in the ferromagnetic hysteresis cycle (exchange bias field $\mathrm{H}_{\mathrm{eb}}$ ) and/or an increase in the ferromagnetic coercive field (exchange enhancement). While exchange bias is usually

a)E-mail: beatrice.negulescu@univ-tours.fr attributed to the pinned uncompensated interfacial spins in the antiferromagnetic structure, the coercive field increase comes from the antiferromagnetic unpinned uncompensated interfacial spins. ${ }^{12}$ In magnetoelectric antiferromagnetic materials, the electric field can control the antiferromagnetic domain structure and consequently the exchange bias effect. ${ }^{13,14}$ Indeed, in $\mathrm{BiFeO}_{3}$ (BFO) based structures, reversible switching between two stable $\mathrm{H}_{\mathrm{eb}}$ values upon polarization reversal was measured in $(\mathrm{La}, \mathrm{Sr}) \mathrm{MnO}_{3} / \mathrm{BFO}$ heterostructures below $30 \mathrm{~K},{ }^{15}$ while a non-reversible room temperature electric field modulation of $\mathrm{H}_{\mathrm{eb}}$ was evidenced in $\mathrm{CoFeB} / \mathrm{BFO}$ based heterostructures. ${ }^{16}$ Finally, a room temperature reversible rotation of the magnetic anisotropy axis between two directions was reported in $\mathrm{Co} / \mathrm{BFO}$ samples and attributed to interlayer exchange coupling combined with a particular ferroelectric domain structure, although without exchange bias. ${ }^{17}$

The coexistence of multiple mechanisms is expected in magnetoelectric heterostructures, and their relative importance depends on the thickness of the ferromagnetic layer ${ }^{3}$ as well as on the symmetry of the ferroelectric phase. ${ }^{18}$

We report here on the room temperature control of the magnetic coercive field of $\mathrm{Ni}$ for $\mathrm{Ni} / \mathrm{BFO}$ based thin film heterostructures by using DC electric fields. The voltage driven magnetic coercive field modulation is nonvolatile, reversible, and cyclable, and so, it could be used as a building block for some low-power and electrically switchable spintronic applications.

Epitaxial $\mathrm{BiFe}_{0.95} \mathrm{Mn}_{0.05} \mathrm{O}_{3}$ (BFMO) thin films were deposited on (110)-oriented $\mathrm{GdScO}_{3}$ (GSO) substrates $\left[(001)_{\mathrm{pc}}\right.$ in pseudo cubic notation used from now on in this article] using pulsed laser deposition at a substrate temperature of $700^{\circ} \mathrm{C}$ in an oxygen pressure of $0.2 \mathrm{mbar}$ and a laser fluence of about $2 \mathrm{~J} \mathrm{~cm}^{-2}$. A buffer layer of conducting $\mathrm{La}_{0.8} \mathrm{Sr}_{0.2} \mathrm{MnO}_{3}$ (LSMO) was used as a bottom electrode for electrical measurements. Ferromagnetic $\mathrm{Ni}$ pads protected with $\mathrm{Au}$ were deposited by e-beam evaporation using electron lithography and lift-off processes.

The magnetic properties of circular Ni pads (diameter of $20 \mu \mathrm{m}$ ) were measured with a magneto-optic Kerr effect (MOKE) in the longitudinal mode while applying an out-of- 
(a)

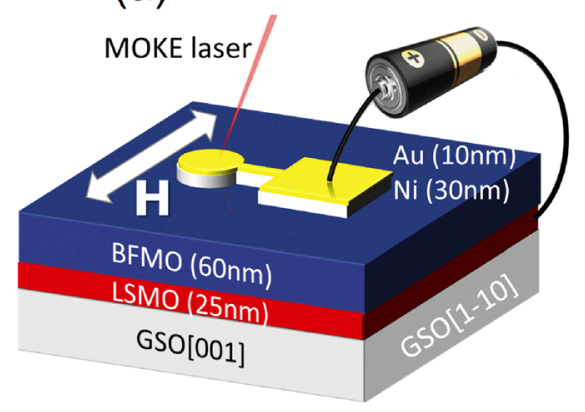

(d)

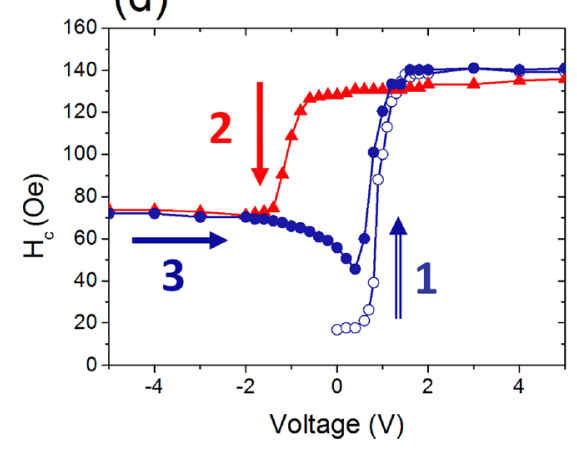

(b)

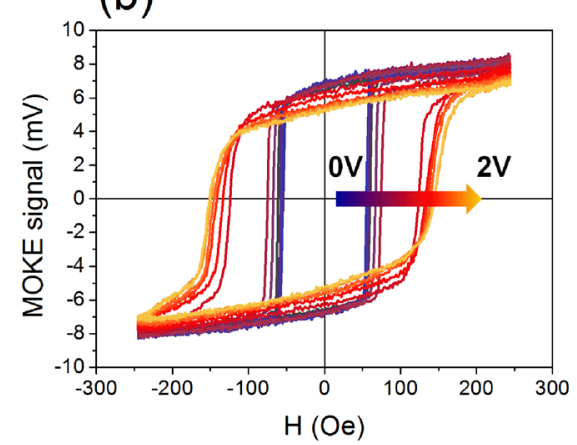

(e)

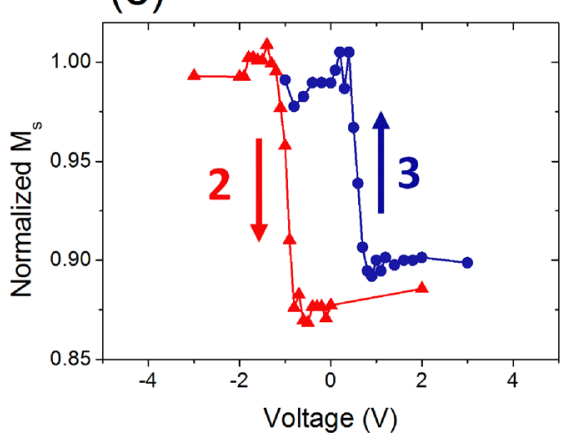

(c)

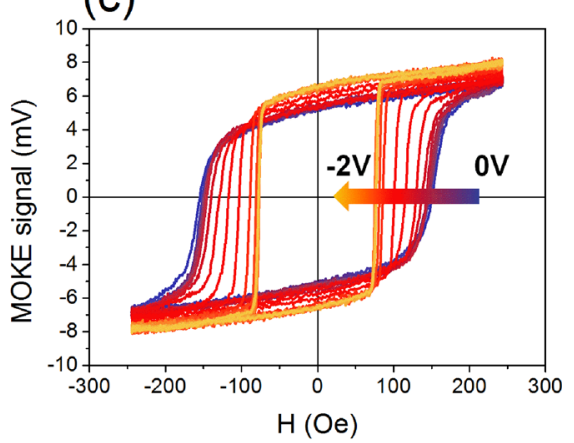

(f)

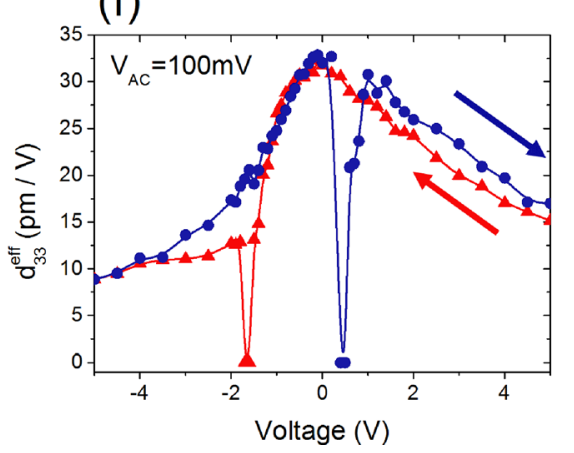

FIG. 1. (a) Schematic of the device and the measurement geometry. (b) MOKE signal as a function of applied magnetic field for increasing voltage from $0 \mathrm{~V}$ to $2 \mathrm{~V}$ (the arrow shows the direction of increasing positive voltage); (c) MOKE signal as a function of applied magnetic field for decreasing voltage from $0 \mathrm{~V}$ to $-2 \mathrm{~V}$ (the arrow shows the direction of increasing negative voltage). (d) Magnetic coercive field as a function of voltage for a complete voltage sweep. (e) Normalized magnetization as a function of voltage. (f) Effective piezoelectric d33 loop as a function of the DC voltage sweep.

plane electric field through the structure. The piezoelectric response of the BFMO films was measured using a double beam laser interferometer, and the ferroelectric domain structure was deduced from piezoresponse force microscopy (PFM). The symmetry and strain state of the films were checked by X-ray diffraction (XRD) with standard (00L) scans and out-of-plane reciprocal space maps around the $(002)_{\mathrm{pc}}$ and (103) $)_{\mathrm{pc}}$ Bragg reflections. The films were found to be partially relaxed with a monoclinic symmetry (see supplementary material). Microstructural characterizations were performed using Transmission Electronic Microscopy (TEM) on cross-sections prepared by a Focused Ion Beam. The high resolution TEM images (supplementary material) show a coherent growth of the LSMO and BFMO oxide layers on the GSO substrate, while the Ni layer was found to be nanocrystalline.

Figure 1(a) shows a schematic of the device as well as the geometry of the measurement. Figures $1(\mathrm{~b})$ and 1 (c) present the magnetic hysteresis cycles for different electric field strengths: (b) with increasing positive voltage and (c) with increasing negative voltage. From Fig. 1(b), a rapid increase in the magnetic coercive field $\left(\mathrm{H}_{\mathrm{c}}\right)$ from about $50 \mathrm{Oe}$ at $0 \mathrm{~V}$ to a maximum value of about $140 \mathrm{Oe}$ at $2 \mathrm{~V}$ can be observed for voltage values above $0.6 \mathrm{~V}$. The increase in the coercive field is associated with a reduction in the saturation magnetization $\left(\mathrm{M}_{\mathrm{s}}\right)$ and in the remanent magnetization $\left(\mathrm{M}_{\mathrm{r}}\right)$, keeping the ratio $\mathrm{M}_{\mathrm{r}} / \mathrm{M}_{\mathrm{s}}$ constant. Figure 1(c) shows the lowering of $\mathrm{H}_{\mathrm{c}}$ to about $70 \mathrm{Oe}$ when gradually reverting the polarity of the applied electric field. Note that $\mathrm{H}_{\mathrm{c}}$ is about $20 \mathrm{Oe}$ in the unpoled state and that no exchange bias is observed in these measurements.

Figure 1(d) shows the variation of $H_{c}$ for a voltage sweep in the range of $\pm 7 \mathrm{~V}$ including the initial poling.
From the initial state, a $600 \%$ increase in $\mathrm{H}_{\mathrm{c}}$ is obtained (from $20 \mathrm{Oe}$ to about $140 \mathrm{Oe}$ ) with a saturation of the effect for voltage values above $1.5 \mathrm{~V}$. A "high" $\mathrm{H}_{\mathrm{c}}$ state $(130 \mathrm{Oe})$ is maintained when reducing the voltage back to $0 \mathrm{~V}$, showing the remanence of the effect. Increasing negative voltage to about $-1.5 \mathrm{~V}$ reduces the $\mathrm{H}_{\mathrm{c}}$ of $\mathrm{Ni}$ to a "low" state of about $70 \mathrm{Oe} . \mathrm{H}_{\mathrm{c}}$ is further reduced to about $55 \mathrm{Oe}$ when the voltage is brought back to $0 \mathrm{~V}$, so keeping $\Delta \mathrm{H}_{\mathrm{c}}=75 \mathrm{Oe}(135 \%)$ with $\Delta \mathrm{H}_{\mathrm{c}}=\mathrm{H}_{\mathrm{c}}($ high $)-\mathrm{H}_{\mathrm{c}}($ low $)$.

Figure 1(e) shows the normalized magnetization values as a function of the applied voltage. Two distinct saturation states are observed, corresponding to the two coercive states. The change from the low to the high coercive state is associated with a lowering of $\mathrm{M}_{\mathrm{s}}$ of about $12 \%$.

Figure 1(f) shows the effective piezoelectric coefficient $\mathrm{d}_{33}^{\text {eff }}$ as a function of the applied DC voltage. In these measurements, the $\mathrm{d}_{33}^{\text {eff }}$ drop to zero occurs at the ferroelectric coercive field. This confirms that the effect of the voltage on $\mathrm{H}_{\mathrm{c}}$ is not dominated by the piezoelectric properties of BFO but rather by the orientation of the ferroelectric polarization.

To further investigate the origin of the observed $\mathrm{H}_{c}$ modulation, a sample with a continuously varying BFMO thickness was deposited using a motorized mask synchronized with the laser pulses. The magnetic hysteresis loops were then measured for applied DC voltages in the range of $\pm 10 \mathrm{~V}$ along the BFMO wedge.

Figure 2(a) shows the extracted $\mathrm{H}_{\mathrm{c}}$ values as a function of the applied voltage for several thicknesses of the BFMO layer. We note an increase in the $\Delta \mathrm{H}_{\mathrm{c}}$ values as well as a variation of the positive switching voltages with the thickness. $\mathrm{H}_{\mathrm{c}}$ (high) values as well as the positive switching electric fields are presented in Fig. 2(b) as a function of BFMO 
(a)

(b)
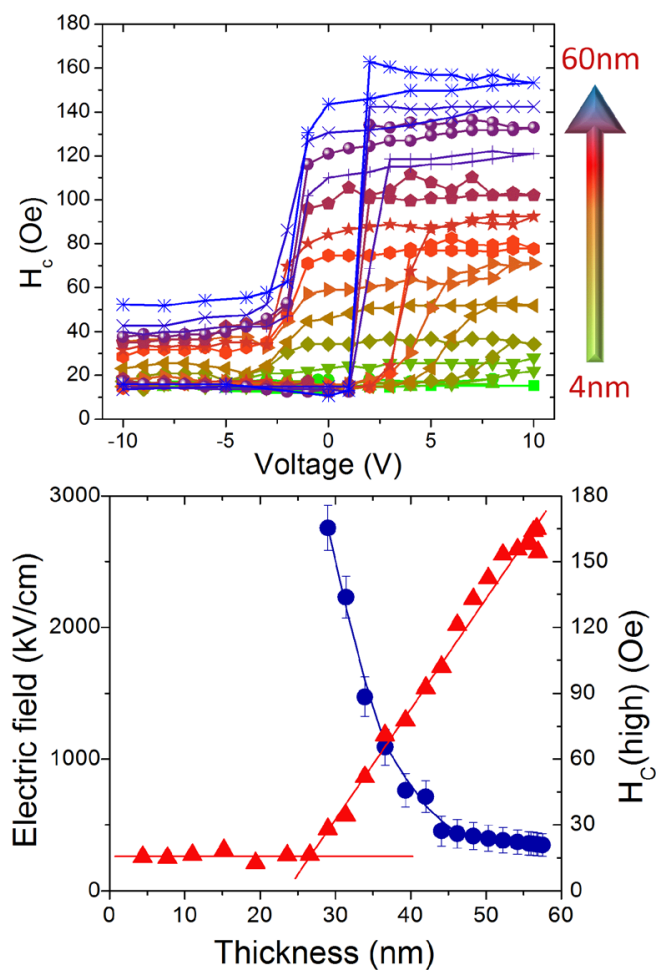

FIG. 2. (a) Magnetic coercive field as a function of voltage for different BFMO thicknesses along the wedge. (b) The variation of the electric field necessary to change the magnetic state as a function of the BFMO thickness (circles) and the coercive field measured at $10 \mathrm{~V}$ as a function of the BFMO thickness (triangles).

thickness. For the thinner part of the wedge, no $\mathrm{H}_{\mathrm{c}}$ variation is found up to an applied voltage of $10 \mathrm{~V}$, showing that there is a critical thickness for the appearance of the effect, determined as about $27 \mathrm{~nm}$. Above this value, a linear increase in the $\mathrm{H}_{\mathrm{c}}$ (high) is observed as a function of thickness. While the positive electric voltage required to induce the "high" $\mathrm{H}_{\mathrm{c}}$ state increases as the thickness is reduced, the negative backswitching field does not seem to be affected by the thickness. A strong imprint is therefore present in the thinner part of the sample, due to the increase in both the built-in field and the depolarizing field with the decrease in the thickness. ${ }^{26}$ All these observations support the conclusion that the effect of the voltage on $\mathrm{H}_{\mathrm{c}}$ modulation is related to the electric polarization switching in the BFMO layer.

The repeatability of the magnetoelectric effect was studied by alternatively switching the polarity of the electrical field for about 500 times. Figure 3 shows the saturation magnetization, the coercive field, and the applied voltage as a function of the number of electric switching events (each event corresponding to an applied voltage of $+2 \mathrm{~V}$ or $-2 \mathrm{~V}$ ). The difference between the two magnetic states slowly decreases with the number of cycles and stabilizes to a value of $\Delta \mathrm{H}_{\mathrm{c}}=47 \mathrm{Oe}$. Again, the same trend is observed for the saturation magnetization that decreases from $12 \%$ to $10 \%$ difference between the two states. The remanence of the effect was also confirmed after the 250 voltage cycles by measuring the $\mathrm{H}_{\mathrm{c}}(0 \mathrm{~V})$ in the two magnetic states $\left(\Delta \mathrm{H}_{\mathrm{c}}\right.$ $=55 \mathrm{Oe})$. The reversibility of this magnetoelectric effect could be used in low power magnetic memories as a potential write assist, similar to heat assisted magnetic recording but easier to achieve. ${ }^{3}$

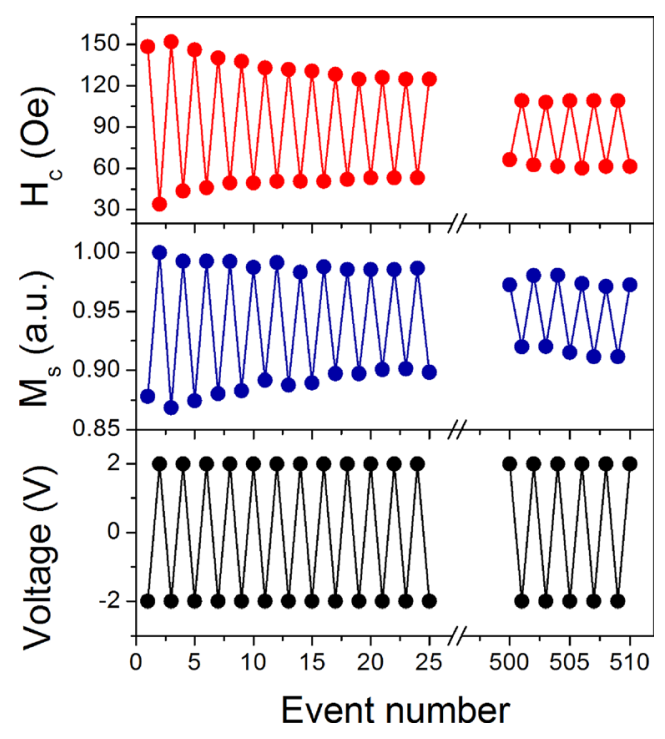

FIG. 3. Top to bottom: coercive field, saturation magnetization, and applied voltage as a function of the event number.

As several mechanisms could be responsible for the studied magnetoelectric effect, we will now discuss the possible origins. From Fig. 1(e), a loop-like dependence of the magnetization versus applied electric field is observed, contrary to the butterfly shape observed for the strain induced effect during major loops. ${ }^{1-3}$ This allows us to discard strain mediated mechanisms to be dominant in our system.

In a recent work, Gao et al. reported the modulation of the magnetic coercive field of $\mathrm{Co}$ in an exchange coupled $\mathrm{Co} / \mathrm{BFO}$ structure. ${ }^{17}$ Owing to the parallelism between the projection of the polarization and the projection of the canted antiferromagnetic moments of BFO, as well as the presence of ferroelectric stripe domains, a magnetic anisotropy was induced in the system and was reversibly rotated by $45^{\circ}$ when switching the ferroelectric polarization back and forth. The PFM images of our BFMO films in the as-deposited state show a mosaic-like structure with no preferential inplane orientation of the polarization [Fig. 4(a)]. As a result, no anisotropy axis could be induced in the $\mathrm{Ni}$ film due to exchange coupling. Furthermore, the electric field does not modify the isotropic state of the Ni film, as indicated by the polar plots of $\mathrm{M}_{\mathrm{r}} / \mathrm{M}_{\mathrm{s}}$ and $\mathrm{H}_{\mathrm{c}}$ presented in Fig. 4(b). We can thus conclude that $\mathrm{H}_{\mathrm{c}}$ modulation in the studied system is not generated by a magnetic anisotropy axis rotation but could correlate with the intrinsic magnetoelectric effect in BFO at the ferroelectric polarization reversal, transferred to $\mathrm{Ni}$ by interface exchange coupling. ${ }^{19}$ Note that similar results to the work of Gao et al. were also found in one of our samples presenting ferroelectric stripe domains (not shown here) so that exchange coupling is also present in our system.

Since we established that the $\mathrm{H}_{\mathrm{c}}$ modulation is related to the ferroelectric polarization reversal, the contribution of the spin-polarized screening charges to the studied magnetoelectric effect should be considered. This mechanism was demonstrated up to now only in ultrathin samples ${ }^{20-22}$ as the Thomas-Fermi screening length is only a few monolayers for metals. A new type of charge mediated coupling effect was recently demonstrated using ferromagnetic resonance 
(a)
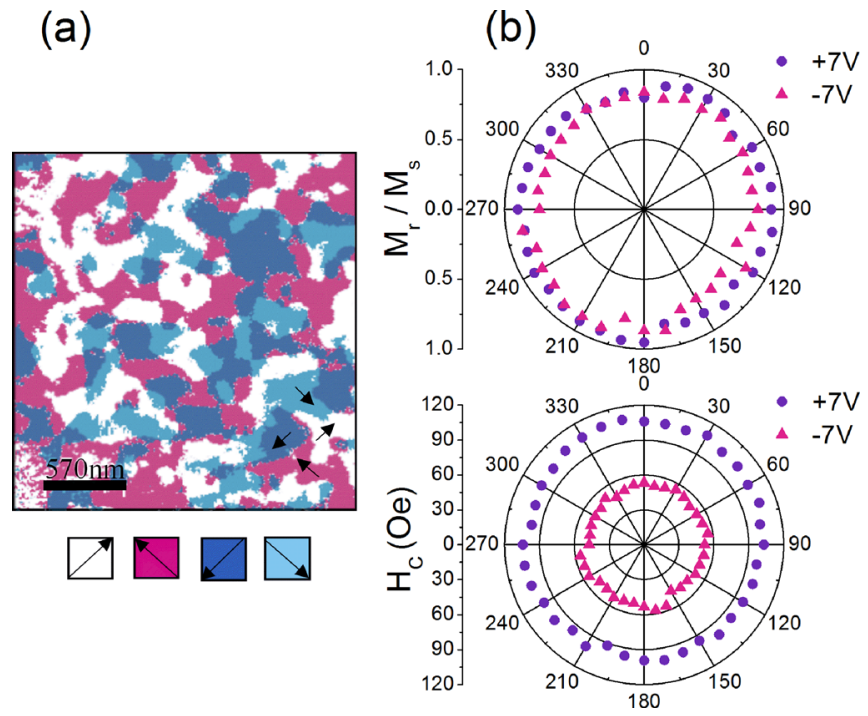

FIG. 4. (a) Reconstructed in-plane PFM phase image using different scan directions in the plane of the sample. The arrows indicate the in-plane projection of the polarization for each domain; (b) $\mathrm{Mr} / \mathrm{Ms}$ (top) and $\mathrm{Hc}$ (bottom) polar plots for two remanent states obtained after applying $\pm 7 \mathrm{~V}$ electric fields.

experiments, defined as a magnon-driven magnetoelectric coupling. Acting within the spin diffusion length, this coupling can drive the magnetic dynamics on thicknesses much larger than the screening length, as demonstrated in $20 \mathrm{~nm}$ in the CoZr film ${ }^{23}$ or $14 \mathrm{~nm}$ in the Co film, ${ }^{24}$ both deposited on PMN-Pt substrates. However, these results obtained from dynamic measurements are not directly transposable to our static experiments. The crystallinity of the magnetic film could also play a role in the studied coupling mechanism. In amorphous $\mathrm{CoFeB}$, it was shown that interface induced anisotropy propagates through the thickness of the film up to $20 \mathrm{~nm}$ (Ref. 25) and the same effect could occur in our nanocrystalline $\mathrm{Ni}$ film.

Besides electron screening, ion migration (e.g., oxygen vacancies) under the electric field could modify the composition of the BFMO film at the interface with the Ni film or oxidize the Ni interface. Preliminary XPS measurements did not indicate compositional differences between the poled and unpoled states, but further in-depth chemical analysis is necessary in order to detect any redox reaction or ionic migration at the interface. Because Mn doping could be the source of charged defects, an undoped BFO film was grown and measured under the same conditions as for the Mndoped BFO samples. The same modulation of the magnetic coercive state by the electric field was observed in this sample (not shown here), although with a $20 \%$ reduced amplitude when compared with BFMO (averaged over 18 devices for each sample). This result indicates that although it is not at the origin of the magnetoelectric effect, Mn doping enhances it.

To conclude, we studied the effect of the electric field on the magnetic properties in $\mathrm{Ni} / \mathrm{BiFe}_{0.95} \mathrm{Mn}_{0.05} \mathrm{O}_{3}$ magnetoelectric heterostructures grown on single crystal (110) oriented $\mathrm{GdScO}_{3}$ substrates. The magnetic coercive field and the saturation of the Ni film are modulated using moderate DC electric voltages. The intensity of the effect depends linearly on the thickness of the BFMO layer at least up to $60 \mathrm{~nm}$, and a critical thickness of $27 \mathrm{~nm}$ was identified for its appearance. The $H_{c}$ and $M_{s}$ modulation is associated with the ferroelectric polarization reversal and could be induced by the coupling of the BFMO ferroelectric polarization with the spin of the Ni film (charge effects) and by the coupling of the antiferromagnetic structure of BFO with the ferromagnetic Ni structure (exchange coupling effect). Further experiments are needed in order to distinguish between these two coupling mechanisms. The repeatability of the studied magnetoelectric effect was demonstrated up to 500 switching events (250 cycles) at room temperature which is promising for applications in the field of spintronics.

See supplementary material for the XRD and TEM structural characterization of the films.

This work was funded by Région Centre in the frame of the project Comhet. The authors would like to thank Sylvain Roger for the target synthesis, Thierry Le Mogne for XPS measurements, and Antoine Ruyter for useful discussions.

${ }^{1}$ V. Garcia, M. Bibes, and A. Barthélémy, C. R. Phys. 16, 168 (2015).

${ }^{2}$ C. Song, B. Cui, J. Peng, H. Mao, and F. Pan, Chin. Phys. B 25, 067502 (2016).

${ }^{3}$ J.-M. Hu, L.-Q. Chen, and C.-W. Nan, Adv. Mater. 28, 15 (2016).

${ }^{4}$ J. Wang, J. Ma, Z. Li, Y. Shen, Y. Lin, and C. W. Nan, J. Appl. Phys. 110, 043919 (2011).

${ }^{5}$ C. Thiele, K. Dörr, O. Bilani, J. Rödel, and L. Schultz, Phys. Rev. B 75, 054408 (2007).

${ }^{6}$ S. Sahoo, S. Polisetty, C.-G. Duan, S. S. Jaswal, E. Y. Tsymbal, and C. Binek, Phys. Rev. B 76, 092108 (2007).

${ }^{7}$ T. Nan, Z. Zhou, M. Liu, X. Yang, Y. Gao, B. A. Assaf, H. Lin, S. Velu, X. Wang, H. Luo, J. Chen, S. Akhtar, E. Hu, R. Rajiv, K. Krishnan, S. Sreedhar, D. Heiman, B. M. Howe, G. J. Brown, and N. X. Sun, Sci. Rep. 4, 3688 (2014).

${ }^{8}$ U. Bauer, L. Yao, A. J. Tan, P. Agrawal, S. Emori, H. L. Tuller, S. van Dijken, and G. S. D. Beach, Nat. Mater. 14, 174 (2015).

${ }^{9}$ D. A. Gilbert, A. J. Grutter, E. Arenholz, K. Liu, B. J. Kirby, J. A. Borchers, and B. B. Maranville, Nat. Commun. 7, 12264 (2016).

${ }^{10}$ X. Zhu, J. Zhou, L. Chen, S. Guo, G. Liu, R.-W. Li, and W. D. Lu, Adv. Mater. 28, 7658 (2016).

${ }^{11}$ S.-X. Ren, L.-Y. Zhang, J.-Y. Dong, Y.-F. Huang, J.-J. Guo, L. Zhang, J. Zhao, X. Zhaoa, and W. Chen, J. Mater. Chem. C 3, 4077 (2015).

${ }^{12}$ H. Béa, M. Bibes, F. Ott, B. Dupé, X.-H. Zhu, S. Petit, S. Fusil, C. Deranlot, K. Bouezhouane, and A. Barthélémy, Phys. Rev. Lett. 100, 017204 (2008).

${ }^{13}$ X. He, Y. Wang, N. Wu, A. N. Caruso, E. Vescovo, K. D. Belashchenko, P. A. Dowben, and C. Binek, Nat. Mater. 9, 579 (2010).

${ }^{14}$ T. Zhao, A. Scholl, F. Zavaliche, K. Lee, M. Barry, A. Doran, M. P. Cruz, Y. H. Chu, C. Ederer, N. A. Spaldin, R. R. Das, D. M. Kim, S. H. Baek, C. B. Eom, and R. Ramesh, Nat. Mater. 5, 823 (2006).

${ }^{15}$ S. M. Wu, S. A. Cybart, D. Yi, J. M. Parker, R. Ramesh, and R. C. Dynes, Phys. Rev. Lett. 110, 067202 (2013).

${ }^{16}$ J. Allibe, S. Fusil, K. Bouzehouane, C. Daumont, D. Sando, E. Jacquet, C. Deranlot, M. Bibes, and A. Barthelémy, Nano Lett. 12, 1141 (2012).

${ }^{17}$ T. Gao, X. Zhang, W. Ratcliff, S. Maruyama, M. Murakami, A. Varatharajan, Z. Yamani, P. Chen, K. Wang, H. Zhang, R. Shull, L. A. Bendersky, J. Unguris, R. Ramesh, and I. Takeuchi, Nano Lett. 17, 2825 (2017).

${ }^{18}$ Y. Liu, Y. Zhao, P. Li, S. Zhang, D. Li, H. Wu, A. Chen, Y. Xu, X. F. Han, S. Li, D. Lin, and H. Luo, ACS Appl. Mater. Interfaces 8, 3784 (2016).

${ }^{19}$ J. T. Heron, D. G. Schlom, and R. Ramesh, Appl. Phys. Rev. 1, 021303 (2014).

${ }^{20}$ W.-G. Wang, M. Li, S. Hageman, and C. L. Chien, Nat. Mater. 11, 64 (2012). 
${ }^{21}$ Y. Shiota, T. Nozaki, F. Bonell, S. Murakami, T. Shinjo, and Y. Suzuki, Nat. Mater. 11, 39 (2012).

${ }^{22}$ Z. Zhou, B. M. Howe, M. Liu, T. Nan, X. Chen, K. Mahalingam, N. X. Sun, and G. J. Brown, Sci. Rep. 5, 7740 (2015).

${ }^{23}$ C. Jia, F. Wang, C. Jiang, J. Berakdar, and D. Xue, Sci. Rep. 5, 11111 (2015).
${ }^{24}$ C. Zhou, L. Shen, M. Liu, C. Gao, C. Jia, and C. Jiang, Phys. Rev. Appl. 9, 014006 (2018).

${ }^{25}$ A. T. Hindmarch, A. W. Rushforth, R. P. Campion, C. H. Marrows, and B. L. Gallagher, Phys. Rev. B 83, 212404 (2011).

${ }^{26}$ Y. Liu, X. Lou, M. Bibes, and B. Dkhil, Phys. Rev. B 88, 024106 (2013). 